\title{
THE PITFALL OF ORAL MUCOCUTANEUS CANDIDA PARAPSILOSIS IN A CHILD WITH HIV INFECTION
}

Leny Kartina, Dwiyanti Puspitasari, Dominicus Husada, Parwati Setiono Basuki, Ismoedijanto

Department of Child Health Faculty of Medicine, University of Airlangga/Dr. Soetomo Hospital

Surabaya - Indonesia

\section{BACKGROUND}

- Candida parapsilosis is an emerging and leading cause of invasive candida infection.

- Children with immunodeficiency including HIV infection have the highest risk for this infection.

- Candidiasis is the most frequent opportunistic fungal infection among patients infected with human immunodeficiency virus.

- It has been estimated that more than $90 \%$ of HIV-infected patients develop this often debilitating infection at some time during the progression of their disease, although the incidence of this disease in HIV infection has been significantly reduced since the introduction oh highly active antiretroviral therapy (HAART)

- Mucocutaneus candidiasis is frequent manifestation in HIV children that often met difficult in diagnostic and management.

- A multidisciplinary approach like surgical debridement, pathologica anatomy, clinical microbiology, and medical treatment is needed to lead a good outcome.

- The purpose of this case is to report the first case of oral mucocutaneus Candida parapsilosis in pediatric patient with HIV infection focusing on its diagnosis approach and management.

\section{CASE REPORT}

A 3 years old HIV boy, good nutrition, and under antiretroviral treatment came with chronic wound perioral with black crust that occur since one month after felt down from his bike. The wound became wider even got previous treatment. He looked pale, no dyspnea and fever.

He treated with many antibiotics (ampicillin, gentamisin, meropenem) based on Klebsiella pneumonia and Acinetobacter baumanii sensitivity from wound swab culture without significant improvement.
DISCUSSION

The skin biopsy revealed suspicious of squamous cell carcinoma. It resulted a doubt because it did not match to clinical presentation of malignancy. The wide excision debridement and open biopsy had been done and the culture revealed candida parapsilosis sensitive to fluconazole. The CD4 count evaluated was very low 30 (1.9\%). Antibiotics treatment then was stopped. Fluconazole was administered, and ART was switched to $2^{\text {nd }}$ line.

After 14 days of fluconazole treatment, the wound fully recovered the perioral crust was detached and left the hypopigmentation macula and good condition.

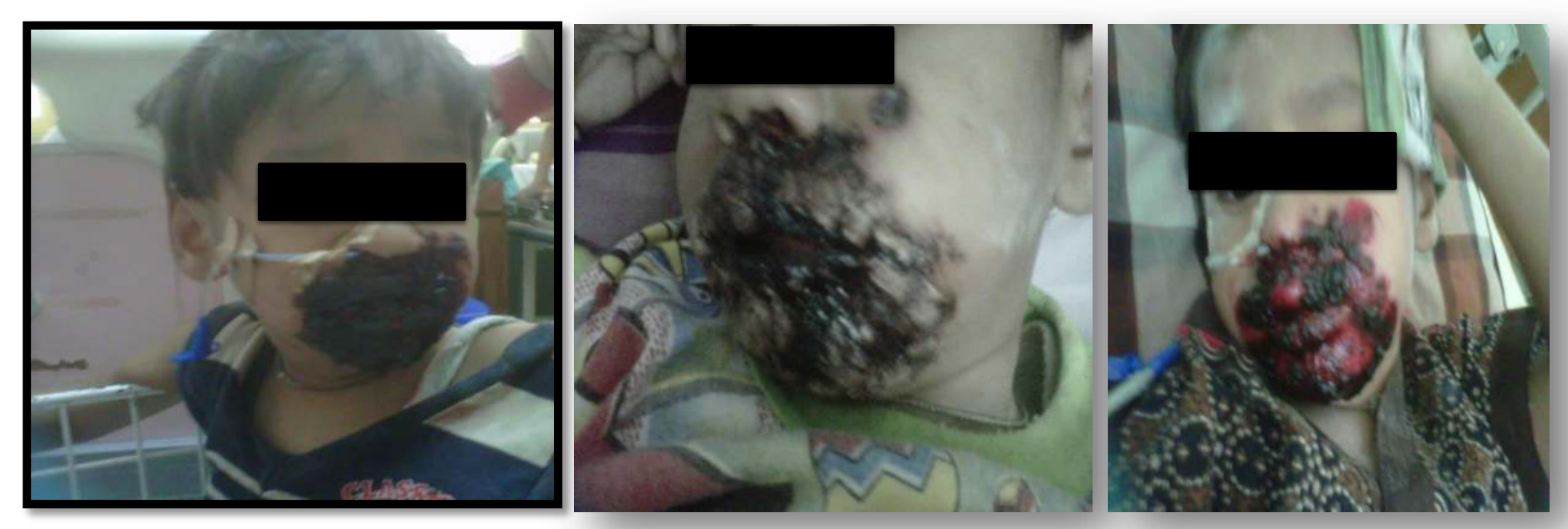

Fig 1. The crust were exfoliated, the bottom of crust look pink and reddish with continous bleeding

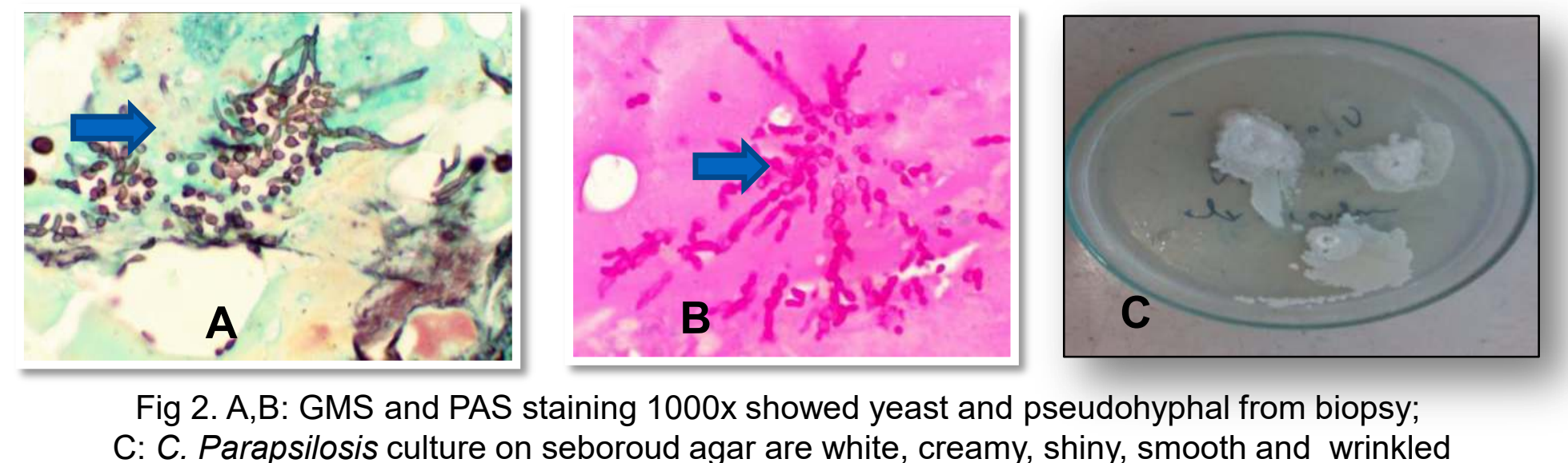

Facing a child with HIV infection and perioral lession, a thought about predisposition infection may be associated with his imunological status. The perioral lession in HIV patient can caused by many condition such as viral infection, bacterial infection, fungal infection and malignancy.

There were diagnostic procedures had been indicated for finding the primary causes and multidisciplinary approach like surgical debridement, pathological anatomy, clinical microbiology, and medical treatment is needed.

Low CD4 and candidiasis has considerable clinical significance in the prognosis of HIV infection and as an indicator of the noneffectiveness of antiretroviral treatment. Switch to second line ART have to be considered. The proper treatment based on fungal culture sensitivity lead the good outcome.

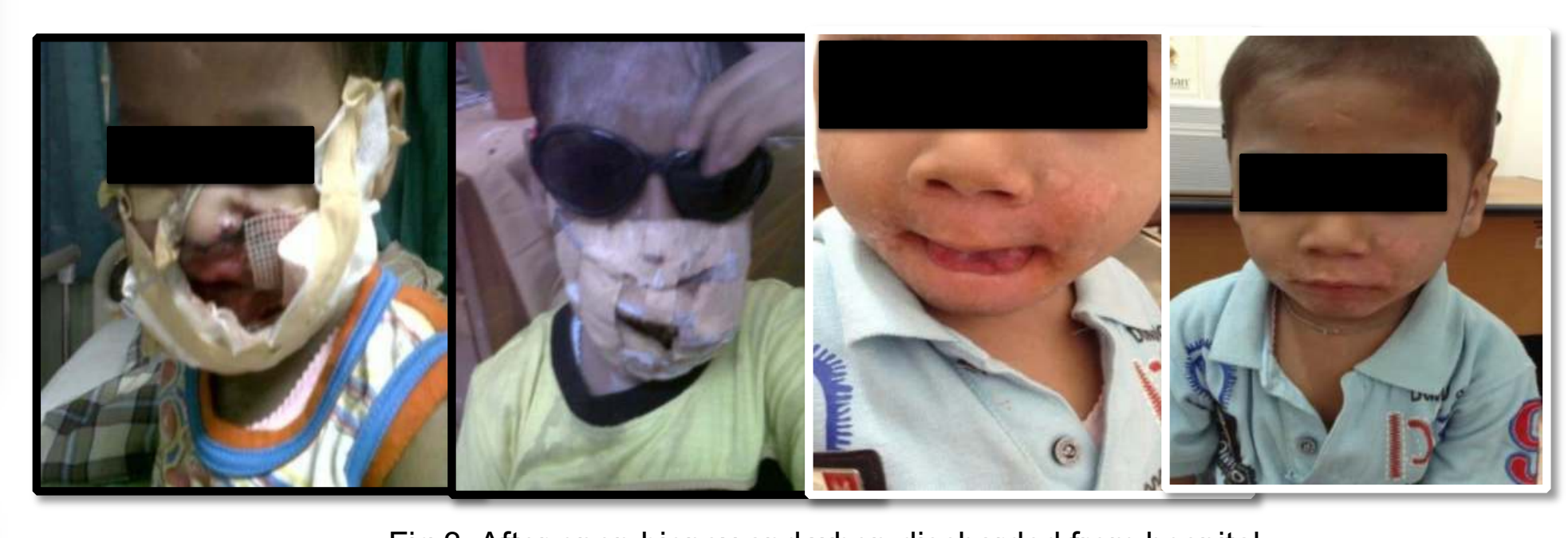

Fig 3. After open biopsy and when discharded from hospital

LEARNING POINT

Candida infection has to be considered in chronic wound in immunocompromised condition such as HIV patients. Surgical debridement, microbiological examination, antifungal and review of previous treatment to consider the resistance of $1^{\text {st }}$ line ARV led a good outcome, highlighting the need for multidiscipline approach. 